PROBLEMS OF ENGINEERING CYBERNETICS AND ROBOTICS • $2021 \bullet$ Vol. 75, pp. 43-50

p-ISSN: 2738-7356; e-ISSN: 2738-7364

https://doi.org/10.7546/PECR.75.21.05

\title{
Incremental Sinusoidal Approximation of Time Series with LibreOffice Calc Solver
}

\section{Veneta Velichkova, Petar Tomov, and Todor Balabanov}

Institute of Information and Communication Technologies

at the Bulgarian Academy of Sciences

Acad. Georgi Bonchev Str., block 2, office 514, 1113 Sofia, Bulgaria

veneta.velichkova@iict.bas.bg,petar.tomov@,iict.bas.bg,todor.balabanov@iict.bas.bg

\begin{abstract}
Financial time series forecasting has high importance in many decisionmaking situations. There are many methods and approaches for time series forecasting. Artificial Neural Networks are widely used. Support Vector Machines are giving even better results than artificial neural networks. Both tools are pretty complicated and have their advantages and disadvantages. This research proposes an approximation based on cumulative sinusoids. From the Fourier Transform, it is well known that each signal can be represented as a sum of sine functions. Optimal coefficients for the sine functions and the linear component, in this research, are searched with the Solver module of LibreOffice Calc. The search of the optimal values is done on an incremental basis. The result show that the time series can be successfully handled as signals.
\end{abstract}

Keywords: Differential evolution, financial forecasting, particle swarm optimization, time series.

\section{Introduction}

Time series are widely used in the financial world. Time series forecasting has its place in the soft computing state of the art [1]. In many situations, the financial data are presented as values that change with time passing. The prices of the stocks are changing with time (gas and oil for example [2]). The prices of companies' shares are changing with time. The rates between the national currencies are changing with time. Taking financial decisions manly when it comes to investments is very dependent on reliable forecasting which is mathematically 
augmented [3]. In the last two centuries, many forecasting tools and approaches are developed. Econometrics is the branch of science responsible for giving empirical content to economic relationships. Thousands of financial indicators/oscillators are created [4]. Thousands of trading strategies are built around the financial indicators/oscillators. In the last few decades, the algorithms of the field of Artificial Intelligence and Machine Learning are also involved in trading decision making [5]. Artificial Neural Networks [6] and Support Vector Machines [7] became very popular in the field. For example, artificial neural networks can be used for financial time series forecasting implemented in Android service and widgets [8] and a proposed modified support vector machine classifier can be used to forecasting short-term trends on the stock market [9].

All forecasting tools in the field of financial times series forecasting depend on the fact that economic processes depend on what happened in the past periods [10]. This branch of forecasting is known as Technical Analysis [11]. It does not count news. It does not count intuitive relationships. It only counts the exact numbers from the past. Fundamental Analysis [12] is another approach for financial forecasting. It relies on intuitive relationships and empirical experience. Such software components could be reusable via service interfaces realized as Service Oriented Architecture [13]. In addition, some of the processes could be automated by using of proper analysis of requests [14]. This research is concentrated on the Technical Analysis and finding of ways to make forecasting with the tools of mathematics and global heuristic optimization algorithms.

Time series in this research is taken as signals [15] in the temporal domain. The measured values are used for building approximation curves. The hope is that when a particular curve fits the known data it will have some forecasting capabilities for the future unknown values. Finding coefficients for the chosen mathematical formulas is a complex optimization problem. The size of the variable space rises with the model refinement process. This fact makes the optimization problem even harder.

The paper is organized as follows: After the literature review part, the incremental sinusoidal approximation is presented. The theoretical proposal is followed by an experimental section. The paper finishes with conclusions and directions for future work.

\section{Incremental Sinusoidal Approximation}

Fast Fourier Transform is the most used method for signal decomposition met in the literature $[16,17]$. It is fast as time consumption and efficient because it is in the group of the exact numerical methods. Even with these advantages, it does not give an answer to what is the minimum of sine functions to be used in the process 
of interpolation and after that extrapolation. The generalization capability of a sinus approximation tool is closely related to small in number calculations and optimal values for the coefficients used.

Time series are points measured in a temporal order. Using these discrete measures, curves can be fitted according to them. For example, Lagrange Interpolating Polynomial is one of the most popular tools in this field. It is well known that an infinite number of curves can be fitted to a finite number of discrete points. In the field of signal processing, it is a very common curve fitting to be done by the usage of sine functions. In most financial time series there are many ups and downs. Financial time series are perfect candidates for approximation with sine functions. Almost all time series have a linear component known as trend. The trend can be easily approximated with the equation of a line (Eq. 1). The sum of sine functions after that can approximate the ups and downs.

$$
y(t)=B . t+C+A_{1 .} \cdot \sin \left(\omega_{1 .} t+\varphi_{1}\right)+A_{2} \cdot \sin \left(\omega_{2 .} . t+\varphi_{2}\right)+A_{3 .} \cdot \sin \left(\omega_{3 .} t+\varphi_{3}\right)+\ldots
$$

Where $B$ is the slope of the linear component, $C$ is the intercept of the linear component. Also, $A_{n}$ is the amplitude of the $n$ sine function, $\omega_{n}$ is the angular velocity of the $n$ sine function, and $\varphi_{n}$ is the phase of the $n$ sine function. The incremental part of the approximation consists of the fact that the linear component is optimized alone by itself first. All sine amplitudes, angular velocities, and phases are given to zero. When the linear component is estimated the coefficients of the first sine function are optimized. The optimization process stops when there is no more convergence. After the first sine function, the second sine function is optimized, and so on as a kind of hierarchy [18]. Sine functions are added until there is a significant difference in the achieved minimum of the mean square root error. The mean square root error is calculated between time series measures and the approximated values.

\section{Experiments and Results}

All experiments are done on a single processor desktop machine - Intel Core i5, 2.3 GHz, 2 Cores, 8GB RAM with macOS High Sierra 10.13.6 and LibreOffice 7.0.5.2. As financial time series, publicly available rates of Bitcoin to USD is taken on a daily interval (Fig. 1).

The optimization starts with all variable cells full with zeros. In the column, $A$ time is presented as an Excel numerical value for the date. Column $B$ holds the value of Bitcoin for 31 days' period. Column $C$ holds the forecasted value. Column $D$ holds the square root difference between the measured and the forecasted value. 


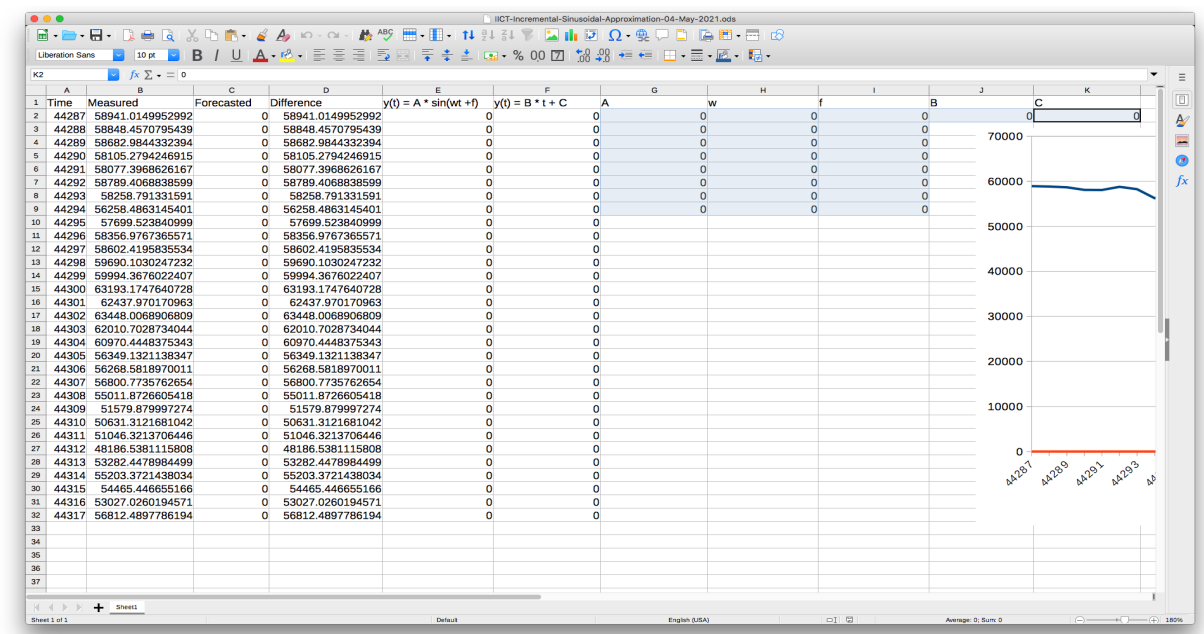

Fig. 1. Initial values as an optimization starting point.

The mean square root error value is accumulated in cell $M 2$ and this is the cell subject to minimization (Fig. 2).

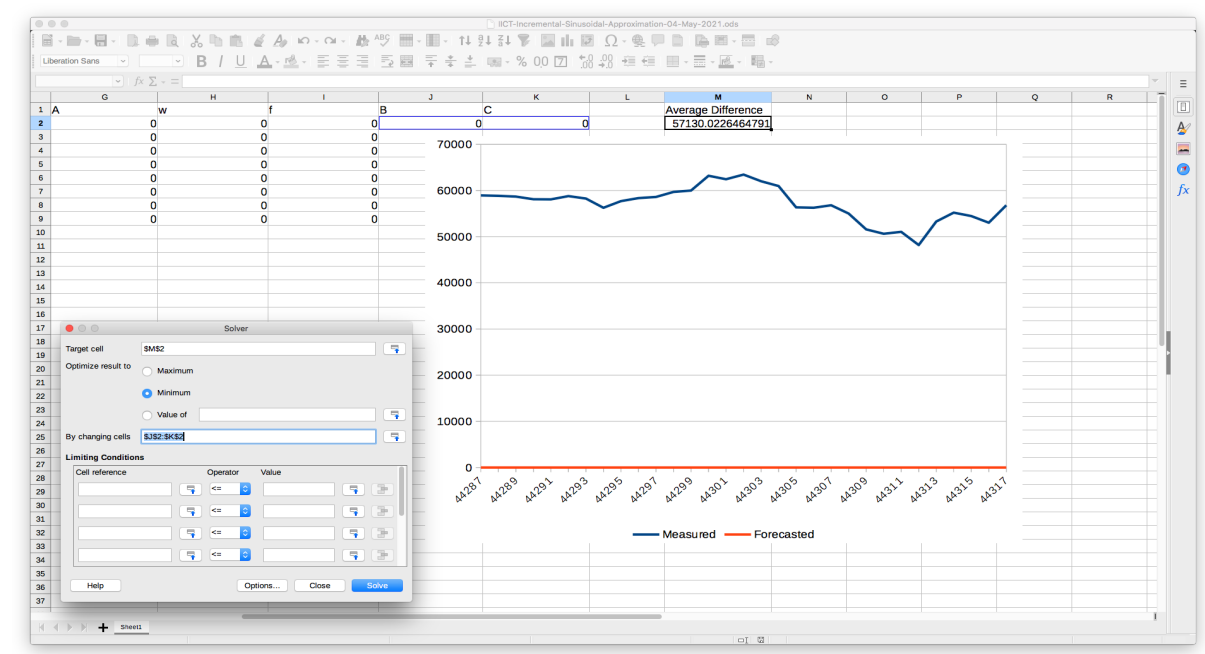

Fig. 2. Start incremental optimization by finding optimal values for the linear component.

Incremental optimization starts with searching for optimal values used as coefficients of the line equation. LibreOffice Calc Solver uses a hybrid implementation of Differential Evolution and Particle Swarm Optimization as optimizers. Coefficients for the linear approximation are found in 10.11 seconds (Fig. 3). 


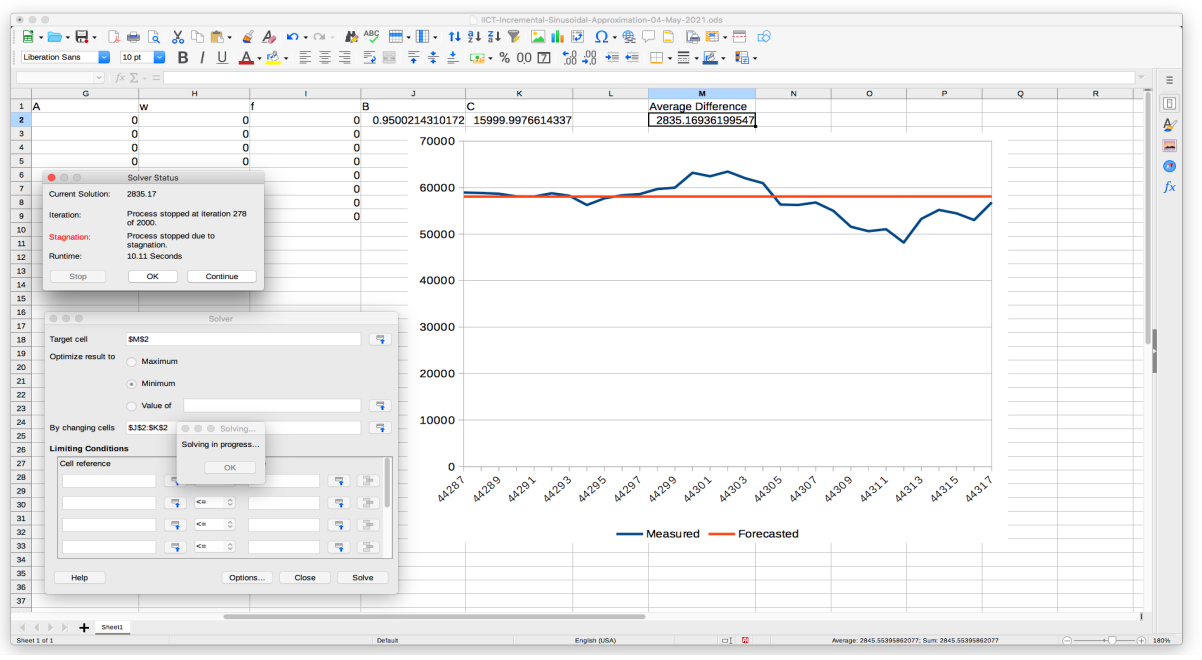

Fig. 3. Coefficients for linear approximation.

After the trend has been estimated, the coefficients for the first sine function are taken for optimization. Stagnation of the optimization with the first sine function is achieved after 150 seconds (Fig. 4).

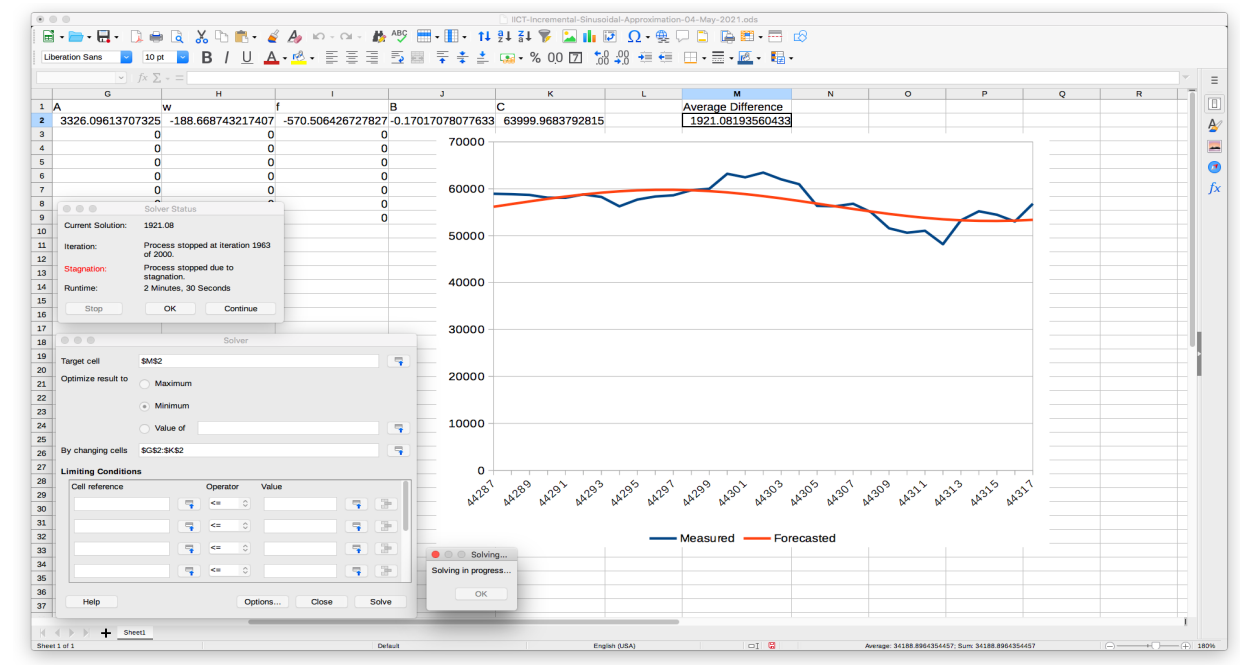

Fig. 4. Coefficients for the first sine function.

After achieving the most accurate possible approximation with a single sine function, the second one is added. Stagnation of the optimization with the two sine functions is achieved after 526 seconds (Fig. 5). 


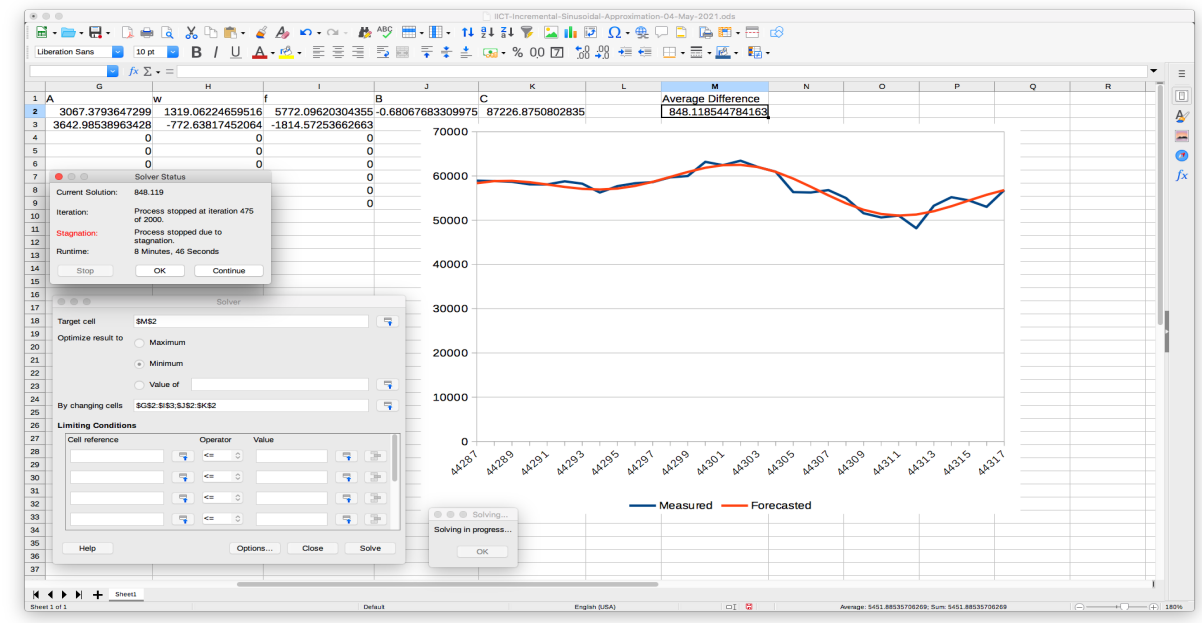

Fig. 5. Coefficients for the second sine function.

Even visually it is clear that a linear component with two sine functions is enough for forecasting with the given input data. Just to prove that two sine functions are enough, a third sine function is added and the optimization target cell is observed that significantly better minimum is difficult to be achieved (Fig. $6)$.

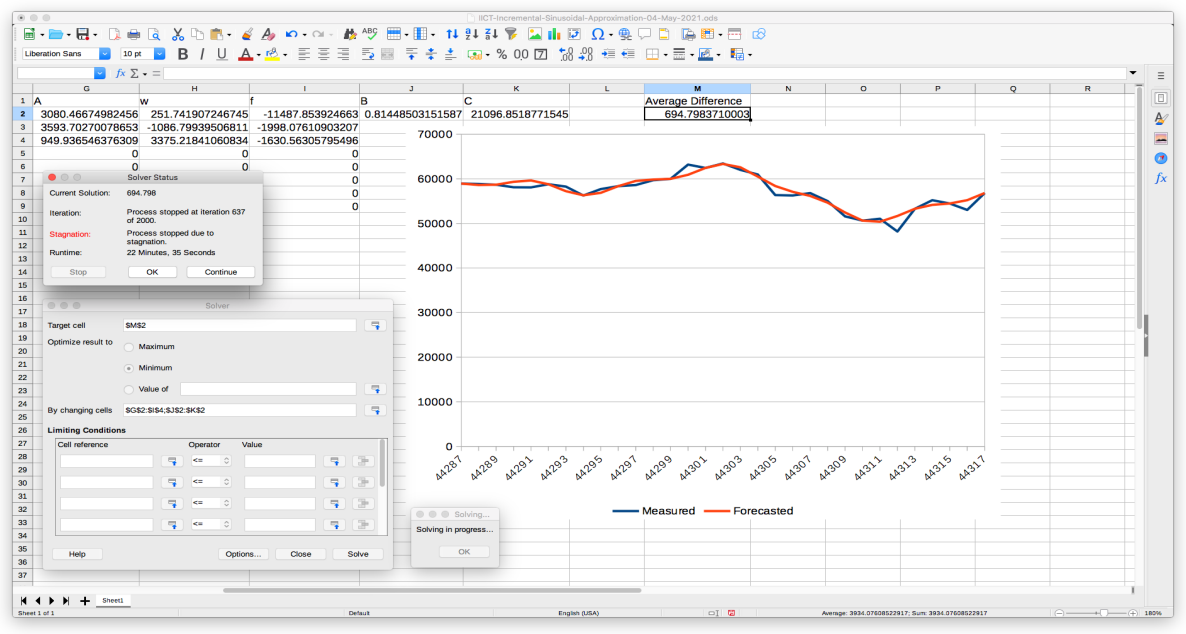

Fig. 6. Coefficients for the third sine function.

Stagnation of the optimization with the three sine functions is achieved after 1355 seconds. The incremental approximation process was stopped on this level because adding too many sine functions can lead to undesired overfitting of the 
model. The time series used in this experiment is relatively short. For more practical usage much bigger time series should be used.

\section{Conclusions}

In this paper, incremental sinusoidal approximation of time series with LibreOffice Calc Solver was proposed. The Solver has a hybrid optimizer based on Differential Evolution and Particle Swarm Optimization. The time series are handled as signals. Coefficients for linear component and sinusoids are found by incremental optimization. The experiments and results show that such a forecasting approach can be very promising.

As further work, some automation as LibreOffice Calc macros would be interesting. Incremental optimization is done manually at this stage (humancomputer interactions) of the research. From a practical point of view, the proposed forecasting can be implemented as service-oriented software architecture.

\section{Acknowledgments}

This work was funded by Velbazhd Software LLC and it was partially supported by the Bulgarian Ministry of Education and Science under the National Research Program Smart Crop Production, approved by Decision of the Ministry Council No.866/26.11.2020.

\section{References}

1. Angelova, V.: Investigations in the area of soft computing targeted state of the art report. Cybernetics and Information Technologies 9(1), 18-24 (2009).

2. Petrov, I: On entropy and concentration analysis of industrial and market systems. In: International Conference on Big Data, Knowledge and Control Systems Engineering Proceedings, pp. 11-23, John Atanasoff Union on Automatics and Informatics, Sofia (2016).

3. Cvetkova, P., Pandulis, A., Borissova, D.: Application of information technologies to support mathematically reasoned decisions. In: Knowledge Society and 21st Century Humanism Proceedings, pp. 488-496, Academic Publishing House Za Bukvite - O Pismeneh, Sofia (2020).

4. Wang, Y., Liu, L., Wu, C.: Forecasting commodity prices out-of-sample: Can technical indicators help? International Journal of Forecasting 36(2), 666-683 (2020).

5. Mankolli E., Guliashki V. (2020) Machine learning and natural language processing: Review of models and optimization problems. In: Dimitrova V., Dimitrovski I. (eds) ICT Innovations 2020. Machine Learning and Applications. Communications in Computer and Information Science, vol. 1316, pp. 71-86 (2020), https://doi.org/10.1007/978-3-030-62098-1 7 
6. Sideratos, G., Ikonomopoulos, A., Hatziargyriou, N: A novel fuzzy-based ensemble model for load forecasting using hybrid deep neural networks. Electric Power Systems Research 178, 106025 (2020).

7. Liagkouras, K., Metaxiotis, K.: Stock market forecasting by using support vector machines. Machine Learning Paradigms. Learning and Analytics in Intelligent Systems 18, 259-271 (2020).

8. Tomov, P., Zankinski, I., Balabanov, T.: Training of Artificial Neural Networks for financial time series forecasting in Android service and Widgets. Problems of Engineering Cybernetics and Robotics 71, 50-56 (2019).

9. Zbikowski, K.: Using volume weighted support vector machines with walk forward testing and feature selection for the purpose of creating stock trading strategy. Expert Systems with Applications 42(4), 1797-1805, (2015), https://doi.org/10.1016/j.eswa.2014.10.001.

10. Stoyanova, K., Guliashki, V. MOEAs Applications in the Finance Area. In Proc. of the 30-th International Conference of Information Technologies (InfoTech 2016), Varna, Bulgaria, pp. 31-41 (2016).

11. Taylor, M., Allen, H.: The use of technical analysis in the foreign exchange market. Journal of International Money and Finance 11(3), 304-314 (1992).

12. Chen, Y., Chen, Y.: A fundamental analysis-based method for stock market forecasting. In: Fourth International Conference on Intelligent Control and Information Processing Proceedings, pp. 354-359, IEEE, Beijing (2013).

13. Alexandrov, A., Monov, V.: Implementation of a service oriented architecture in smart sensor systems integration platform. In: Third International Conference on Telecommunications and Remote Sensing Proceedings, vol. 1, pp. 114-120, Science and Technology Publications, Luxembourg (2014).

14. Bakanova, N., Atanasova, T.: Method for automated analysis of users' requests to service centre of information networks in OIS. Problems of Engineering Cybernetics and Robotics 74, 33-40, (2020), https://doi.org/10.7546/PECR.74.20.04

15. Korenberg, M., Paarmann, L.: Orthogonal approaches to time-series analysis and system identification. IEEE Signal Processing Magazine 8(3), 29-43 (1991).

16. Garvanov, I., Kabakchiev, Ch., Garvanova, M., Borissova, D., Dimitrov, G.: Diffraction models from opaque objects simulated by Fourier Transform. In: Proc. of 9th International Conference on Telecommunications and Remote Sensing, pp. 24-29, (2020). https://doi.org/10.1145/3430116.3430121

17. Stankovic, I., Dakovic, M., Ioana, C.: Decomposition and analysis of signals sparse in the dual polynomial Fourier transform. Microprocessors and Microsystems 63, 209-215 (2018), https://doi.org/10.1016/j.micpro.2018.09.005.

18. Tashev, T., Hristov, H.: Hierarchical Interconnection Modeling in Computer Systems. In: International Scientific Conference Communication, Electronic and Computer Systems Proceedings, pp. 215-220, Sofia (2000). 